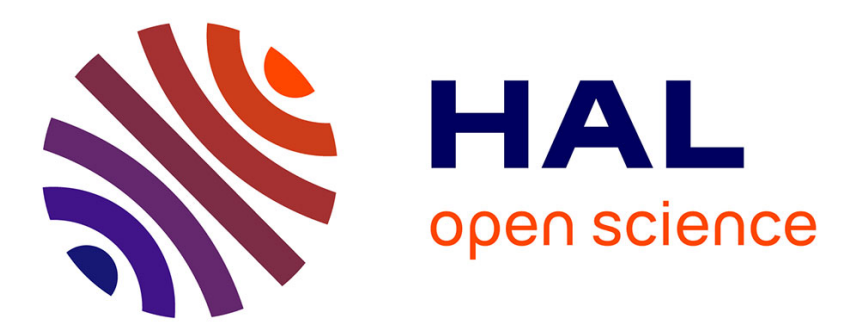

\title{
Stochastic Analysis of UDNs With Resource Capacity and User Scheduling
}

\author{
Yassine Hmamouche, Mustapha Benjillali, Samir Saoudi
}

\section{To cite this version:}

Yassine Hmamouche, Mustapha Benjillali, Samir Saoudi. Stochastic Analysis of UDNs With Resource Capacity and User Scheduling. WCNC 2019: IEEE Wireless Communications and Networking Conference, Apr 2019, Marrakech, Morocco. 10.1109/WCNCW.2019.8902783 . hal-02067730

\section{HAL Id: hal-02067730 https://imt-atlantique.hal.science/hal-02067730}

Submitted on 14 Mar 2019

HAL is a multi-disciplinary open access archive for the deposit and dissemination of scientific research documents, whether they are published or not. The documents may come from teaching and research institutions in France or abroad, or from public or private research centers.
L'archive ouverte pluridisciplinaire $\mathbf{H A L}$, est destinée au dépôt et à la diffusion de documents scientifiques de niveau recherche, publiés ou non, émanant des établissements d'enseignement et de recherche français ou étrangers, des laboratoires publics ou privés. 


\title{
Stochastic Analysis of UDNs With Resource Capacity and User Scheduling
}

\author{
Yassine Hmamouche ${ }^{1,2}$, Mustapha Benjillali ${ }^{2}$, and Samir Saoudi ${ }^{1}$ \\ ${ }^{1}$ IMT Atlantique, Lab-STICC, UBL, 29238 Brest, France \\ Emails: \{yassine.hmamouche; samir.saoudi\}@imt-atlantique.fr \\ ${ }^{2}$ Communication Systems Department, INPT, Rabat, Morocco \\ Email: benjillali@ieee.org
}

\begin{abstract}
In this paper, we investigate the impact of antenna elevation, resource capacity and user scheduling on the performance of ultra-dense networks (UDNs). Using tools from stochastic geometry (SG), we extend a recently introduced definition of coverage probability by inducing a generic thinning that can capture BSs with available resource capacity to transmit users data. Analytical results are then derived for the coverage probability and the average achievable rate, where we obtain closed-form expressions allowing to assess UDNs performance in a more tractable and meaningful fashion compared to the conventional definition of coverage probability. Moreover, we show that the average rate under the new definition requires only the computation of a two-fold numerical integral rather than a four-fold integral in the previous works, which is expected to significantly reduce computational complexity. Comparing the obtained results, we find that the impact of parameters, such as resource capacity, BS transmit power as well as the implementation complexity of scheduling schemes are irrelevant as network density increases, which suggests new insights into the role of these parameters in UDNs.
\end{abstract}

\section{INTRODUCTION}

With the rapidly growing interest in smart-phones and their data-hungry applications, modern cellular networks are increasingly characterized by opportunistic deployment to address end-user specific demands and improve quality of service (QoS) perceptions [1]. Particularly, UDNs based on huge deployment of small-cell BSs are envisioned as the workhorse of capacity improvement in fifth generation $(5 \mathrm{G})$ networks [2].

Accordingly, the analysis and modeling of UDNs require generally powerful mathematical tools and new concepts in order to capture key system parameters that impact the equilibrium of the utility function incorporating users QoS and operators investment. The key challenge in fact, is to develop sufficiently tractable models inducing physically meaningful performance trends. Recently, stochastic geometry (SG) has shown success as a powerful mathematical tool allowing to derive spatial averages of network performance metrics (e.g. coverage probability, average rate,...), and thereby prevents the use of time-consuming computer simulations [3], [4].

Besides, almost all previous studies based on SG models considered the conventional received signal-to-interference-

This work is funded by a research grant from PRACOM and the Regional Council of Brittany, France. plus-noise ratio (SINR) as the key driver for user's quality of experience (QoE). Hence, the complementary cumulative distribution function (CCDF) of SINR, i.e., coverage probability, is in general expressed under an improper integral [3]-[6], requiring efficient and arduous numerical integration [8], except for some special cases where closed-form expressions can be obtained (e.g. path-loss exponent equals four, the interferencelimited regime,...). Furthermore, almost all SG based studies derive the ergodic rate by integrating the coverage probability over the positive real axis, which resorts to a four-fold integral [3, Appendix C], except the Hamdi's lemma based approach presented in [7], which requires only the computation of a twofold numerical integral but needs however the use of Meijer G-function [7, Corollary 1].

To overcome the aforementioned shortcomings, authors of [8], [9] introduced a new definition of coverage probability, where the typical user is in coverage when, i) the user receives a sufficiently good signal strength without any over-provision of the BS transmit power, i.e., the short-term average Signalto-Noise-Ratio $(\overline{\mathrm{SNR}})$ is greater than a certain threshold, ii) the user receives a good signal quality, i.e., the Signal-toInterference-Ratio (SIR) is greater than another threshold. Interestingly, the new framework captures more system-level parameters than the available definition, and enables deriving general closed-form expressions of coverage probability, which is not allowed by the conventional definition.

However, in realistic networks, the user may experience voice/data drops due to congestion at peak demand, regardless of the received signal strength or quality. The critical missing piece in the framework introduced in [8], [9], is then a measure of physical and logical resource capacity on active BSs (e.g., channel resource elements grouped into physical resource blocks (PRB) in 5G New Radio (NR), or the common power channel resource like the P-CPICH channels in UMTS,...).

In this paper, we extend the framework of [8], [9] by tractably capturing BS resource capacity. In this context, we introduce into analysis a generic thinning that can reflect BSs with available capacity. Next, we address for the first time the analysis of UDNs under the new revisited framework of [8], [9]. Typically, we incorporate into analysis three representative scheduling schemes in terms of fairness and implementation complexity. The rationale is to compare their performance in 
UDNs. We also incorporate the BS height since i) its effect is critical in UDNs [10] and ii) to avoid the occurrence of the less-realistic SINR invariance property [3], [6].

The following notation will be used throughout the paper. $\mathbb{P}\{$.$\} and \mathbb{E}\{$.$\} stand for the probability and expectation$ measure. $\mathbb{L}_{X}(s)=\mathbb{E}\left\{\mathrm{e}^{-s X}\right\}$ is the Laplace transform of a random variable $X$ evaluated at $s$. We define for any reals $m, x \in \mathbb{R}, F_{m}(x)={ }_{2} F_{1}(1,-m ; 1-m ;-x)$ where ${ }_{2} F_{1}(., . ; . ; z)$ is the Gauss hypergeometric function, and $\psi^{-1}($. is the inverse function of a function $\psi($.$) .$

\section{SYSTEM MODEL}

\section{A. Cellular Network and Channel Model}

We consider a downlink cellular network, in which the location of BSs and users is modeled with two independent homogeneous PPPs $\Psi_{\mathrm{b}}$ and $\Psi_{\mathrm{u}}$ in the plane $\mathbb{R}^{2}$, with respective densities $\lambda_{\mathrm{b}}$ and $\lambda_{\mathrm{u}}$ measured in $\left[\mathrm{BSs} / \mathrm{m}^{2}\right]$ and [Users $\left./ \mathrm{m}^{2}\right]$, respectively. Without loss of generality, and as permitted by the Slivnyak-Mecke's theorem [4, vol. 1, Theorem 1.4.5], a typical user at the origin $O$, is taken as the object of the analysis.

We assume that all BSs transmit with the same transmit power $P_{\mathrm{tx}}$, and that multipath fading of the link between a BS located at $x_{k} \in \Psi_{\mathrm{b}}$ and the typical user located at $O$, is incorporated by a positive and i.i.d. exponential fading $g_{k}$ with unit mean, while $r_{k} \triangleq\left\|x_{k}\right\|$ denotes the horizontal distance between $x_{k}$ and the typical user such as the subscript 0 and $i$ are used to identify the desired and interfering links, respectively. The radio channel attenuation is dependent on a path-loss function $\ell($.$) , such as the received power at O$ from a BS $x_{k}$ is $P_{\mathrm{rx}}=P_{\mathrm{tx}} g_{k} / \ell\left(x_{k}\right)$. The path-loss function $\ell($.$) is$ assumed to: i) accept an inverse function $\ell^{-1}($.$) , ii) validate the$ dependency condition of $\ell(\sqrt{x y}) / \ell(\sqrt{x})$ on $x \in \mathbb{R}$ for $y \geq 1$ [6, Lemma 1], assumed to avoid the scenario of the SINR invariance property, and iii) $\ell($.$) is a monotonically increasing$ function with distance from the origin, to ensure that the received power cannot exceed the transmitted one. $\sigma^{2}$ denote the variance of the additive noise such as SNR $=P_{\mathrm{tx}} / \sigma^{2}$.

\section{B. Cell Association Model}

We assume the association criterion of the highest average received power, where the typical user is assigned to a unique BS $\left\{x_{0}\right\}$ from $\Psi_{\mathrm{b}}$ such as

$$
x_{0}=\arg \max _{x_{k} \in \Psi_{\mathrm{b}}}\left\{P_{\mathrm{tx}} / \ell\left(r_{k}\right)\right\} \stackrel{(\mathrm{a})}{=} \arg \min _{x_{k} \in \Psi_{\mathrm{b}}}\left\{r_{k}\right\} .
$$

where (a) follows from the property (iii) of $\ell($.$) . The plane$ $\mathbb{R}^{2}$ seen from BSs is then divided into cells corresponding to the spatially nearest points to each BS than to any other BSs of $\Psi_{\mathrm{b}}$, namely the Poisson Voronoi tessellation. We denote by $C_{0}$, the Voronoi cell containing the typical user and the intended BS $x_{0}$, formally named, the Crofton cell [8].

In realistic networks, $x_{0}$ needs to be endowed with available resource capacity to carry the typical user's data. Actually, insufficient capacity is likely due to a bottleneck at the backhaul level and/or the scarcity of traffic channels as a result of high data demand or as a result of maintenance failures (e.g., some channel physical modules are down). At a given time, we denote by $p_{\Theta}$ the probability that a given BS $x_{k}$ has sufficient resource capacity to carry the data of a user requesting connection to $x_{k}$. By construction, $p_{\Theta}$ should differ from the probability $p_{\Delta}$ of having no user associated to $x_{k}$, since the event of the former covers generally that of the latter, and given $p_{\Theta}$ also depends on a parameter $\eta$, correlated to the average inherent resource capacity over all BSs when no user is served. However, both $p_{\Theta}$ and $p_{\Delta}$ need to increase with BS density and shrink with users density. For tractability, we assume that the process $\Theta_{b}$ of BSs with sufficient capacity, is an independent thinning of $\Psi_{\mathrm{b}}$ with density $\lambda_{\Theta}=p_{\Theta} \lambda_{\mathrm{b}}$, where $p_{\Theta}$ will be approximated in the next section.

\section{Scheduling Modeling}

BSs not serving any user are assumed to be in idle mode in order to mitigate the other-cell interference and improve the energy efficiency. Let $\Delta_{\mathrm{b}}$ be the process of BSs in idle mode. The density of $\Delta_{\mathrm{b}}$ is $\lambda_{\Delta}=p_{\Delta} \lambda_{\mathrm{b}}$ where $p_{\Delta}$ is approximated as [11, Proposition 1],

$$
p_{\Delta} \simeq\left(1+\frac{\lambda_{\mathrm{u}}}{3.5 \lambda_{\mathrm{b}}}\right)^{-3.5}
$$

However, on the other hand, when multiple competing users simultaneously need to access the same BS $x_{0}$, three representative scheduling schemes will be considered and investigated to assess the impact on performance in the context of sparse networks and UDNs. An important quantity is the probability mass function (PMF) of the number of competing users inside $C_{0}$. Let $\mathcal{N}_{\mathrm{u}}$ be the random variable that denotes the number of users in $C_{0} \backslash\left\{x_{0}\right\}$. The PMF of $\mathcal{N}_{\mathbf{u}}$ is approximated as [11, Proposition 2],

$$
\mathbb{P}\left\{\mathcal{N}_{\mathrm{u}}=n\right\} \simeq \frac{3.5^{4.5} \Gamma(n+4.5)\left(\lambda_{\mathrm{u}} / \lambda_{\mathrm{b}}\right)^{n}}{\Gamma(4.5) \Gamma(n+1)\left(3.5+\lambda_{\mathrm{u}} / \lambda_{\mathrm{b}}\right)^{n+4.5}} .
$$

Hereafter, the three scheduling schemes considered:

1) Scheduling Model 1 (Non-orthogonal Scheduling): Each BS simultaneously serves multiple users associated with it on the same resource block, this is feasible for example when only the typical user that is associated to $x_{0}\left(\mathbb{P}\left\{\mathcal{N}_{\mathrm{u}}=0\right\}=1\right)$, or when using a non-orthogonal multiple access (NOMA) technique such as power-based NOMA, but at the cost of increased intra-cell interference that may be mitigated by successive interference cancellation (SIC) at the receivers.

2) Scheduling Model 2 (Round-Robin (RR) Scheduling): Simultaneous users are scheduled with equal probability regardless of their channel qualities, which enhance temporal fairness among users and reduce implementation complexity.

3) Scheduling Model 3 (Proportional Fair (PF) Scheduling): Users are scheduled by leveraging their spatial diversity based on the channel quality indicators (CQI), which will increase system throughput at the cost of fairness. For tractability, we assume that the selection of users for downlink transmission based on maximum PF metric is closely reflected by the largest fading gain $g_{k}$, as was endorsed by [12]. 


\section{Network Performance}

Following the limitations mentioned in [8] of the commonly available definition of coverage probability $\mathrm{P}_{\text {cov }}-$ the SINR of the typical user is above a certain threshold-, we consider the new definition adopted in [8], [9], such as the typical user is in coverage when: (i) it receives a sufficiently good signal strength from the nearest BS without any over-provision of the transmitting power $P_{\mathrm{tx}}$, i.e., the average $\overline{\mathrm{SNR}}=$ $P_{\mathrm{tx}} /\left(\sigma^{2} \ell\left(r_{0}\right)\right)=\mathrm{SNR} / \ell\left(r_{0}\right)$ is greater than a threshold $T_{\mathrm{s}}$, ii) the $\mathrm{SIR}=g_{0} P_{\mathrm{tx}} /\left(\ell\left(r_{0}\right) I\right)=g_{0} / \sum_{i} g_{i}\left(\ell\left(r_{0}\right) / \ell\left(r_{i}\right)\right)$ is greater than a threshold $T_{\mathrm{q}}$, where $I$ is the other-cell interference.

The coverage probability will then be expressed as

$$
\mathrm{P}_{\text {cov }}^{(j)}\left(T_{\mathrm{q}}, T_{\mathrm{s}}\right)=\sum_{n=0}^{\infty} \mathbb{P}\left\{\mathrm{SIR} \geq T_{\mathrm{q}}, \overline{\mathrm{SNR}} \geq T_{\mathrm{s}}, \mathcal{N}_{\mathrm{u}}=n\right\},
$$

where the superscript $j=1,2$ or 3 indexes the adopted scheduling model, and we commonly consider that $\mathrm{P}_{\mathrm{cov}}^{(1)}=$ $\mathbb{P}\left\{\mathrm{SIR} \geq T_{\mathrm{q}}, \overline{\mathrm{SNR}} \geq T_{\mathrm{s}}\right\}$, i.e., each BS serves one user.

\section{A. Approximation of the Process of BSs with Available Re- source Capacity}

The resource capacity of a typical BS is closely dependent on the traffic demand from high priority users, which may be the ones with greatest CQI or those privileged through system configuration to access traffic channels (very important users or services). This observation makes it less tractable to derive $p_{\Theta}$ since we do not have a priori knowledge about the distribution of traffic demand from high priority users. To overcome this limitation and approximate $p_{\Theta}$, we assume that the high priority traffic demand is related to the number of users located inside a disc $D_{\mathrm{c}}$ centered at $x_{0}$ and containing: i) sufficiently closest users to $x_{0}$, ii) the closest users from other cells with high probability to make a handover towards $x_{0}$, i.e., the nearest users from the nearest neighboring cell.

Let $\xi_{\mathrm{M}}$ denote the random variable of the radius of the minimal disc $D_{\mathrm{M}}$ centered at $x_{0}$ and containing $C_{0}$, and $\xi_{\mathrm{m}}$ as the radius of the maximal disc $D_{\mathrm{m}}$ centered at $x_{0}$ and contained by $C_{0}$ (see Fig. 1). To the authors knowledge, the distribution of the former does not exist except for some lower bounds as in [13, equation 10] or intractable mathematical expressions as in [14], while the distribution of the latter is totally known as in [13, equation 9]. For analytical tractability, we consider that the radius $\xi_{c}$ of $D_{c}$ is somewhere bounded between $\xi_{m}$ and $\xi_{M}$ such that the disc of radius $\sqrt{\eta} \xi_{c}$ and centered at $x_{0}$ contains at most the nearest $B S$ to $x_{0}$. Formally expressed, we have

$$
\mathbb{P}\left(\xi_{\mathrm{c}}>r\right)=\left(1+\pi \lambda_{\mathrm{b}} \eta r^{2}\right) e^{-\pi \lambda_{\mathrm{b}} \eta r^{2}} .
$$

The PDF of $\xi_{\mathrm{c}}$ is then derived as

$$
f_{\xi_{\mathrm{c}}}(r)=2 \eta^{2}\left(\pi \lambda_{\mathrm{b}}\right)^{2} r^{3} e^{-\pi \lambda_{\mathrm{b}} \eta r^{2}}
$$

and $p_{\Theta}$ is approximated by averaging $\exp \left(-\pi \lambda_{\mathrm{u}} \xi_{c}^{2}\right)$ over the distribution of $\xi_{\mathrm{c}}$, as

$$
p_{\Theta}=\mathbb{E}_{\xi_{\mathrm{c}}}\left\{\exp \left(-\pi \lambda_{\mathrm{u}} \xi_{\mathrm{c}}^{2}\right)\right\}=\left(1+\frac{\lambda_{\mathrm{u}}}{\eta \lambda_{\mathrm{b}}}\right)^{-2} .
$$

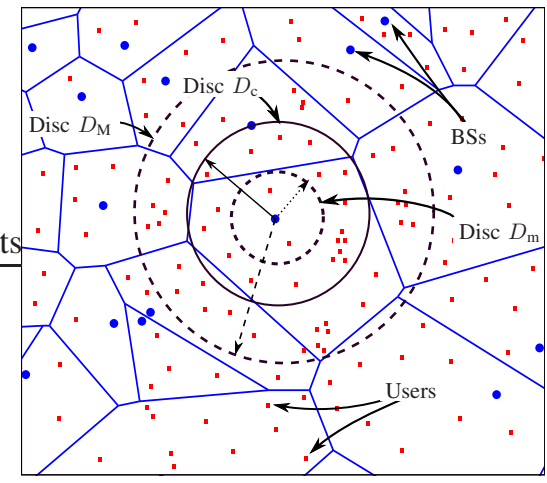

Fig. 1. A PPP realization of BSs and users $\left(\lambda_{\mathrm{u}}>\lambda_{\mathrm{b}}\right)$ where the boundaries of $D_{\mathrm{M}}, D_{\mathrm{m}}$ and $D_{\mathrm{c}}$ are illustrated.

Remark 1. The expression of $p_{\Theta}$ in (7) confirms our initial intuition in Section II. In fact, $p_{\Theta}$ can be increased by acting on the network macroscopic resource capacity via densification with new BSs, or by improving the average intrinsic resource capacity of existing BSs via $\eta$. Besides, increasing users density, will decrease $p_{\Theta}$ and then the availability of BSs to carry users traffic. For fixed densities $\lambda_{b}$ and $\lambda_{u}$, it is obvious to mention from (7) and (2) that, if $\eta$ becomes greater than a threshold $\eta_{0}=\mu\left(\lambda_{u} / \lambda_{b}\right)$, where $\mu(x)=x /\left[(1+x)^{1.75}-1\right]$, the tendency of having more BSs with sufficient capacity increases at the expense of idle mode BSs, which sheds light on the interplay between $p_{\Delta}$ and $p_{\Theta}$. We note also that $\frac{\lambda_{b}}{\lambda_{u}} p_{\Theta}\left(1-p_{\Delta}\right) \rightarrow 1$ as $\lambda_{b} \gg \lambda_{u}$, while $\frac{\lambda_{b}}{\lambda_{u}} p_{\Theta}\left(1-p_{\Delta}\right) \rightarrow 0$ as $\lambda_{u} \gg \lambda_{b}$.

The following lemma describes the scaling law with $\lambda_{\mathrm{b}}$ and $\lambda_{\mathrm{u}}$ of useful combinations of $p_{\Theta}$ and $p_{\Delta}$.

Lemma 1. Under the previous approximations of $p_{\theta}$ and $p_{\Delta}$, we have the following properties:

i) $\frac{\lambda_{b}}{\lambda_{u}} p_{\Theta}\left(1-p_{\Delta}\right)$ scales with $\frac{\lambda_{b}}{\lambda_{b}+\lambda_{u}} \exp \left(-\frac{\theta(\eta) \lambda_{u}}{\lambda_{b}+\lambda_{u}}\right)$, where $\theta(x)$ is a monotonically decreasing function for $x \geq 1$.

ii) For every reals $M, N \geq 0, \frac{1-\exp \left(-M\left[p_{\Theta}+N\left(1-p_{\Delta}\right)\right]\right)}{p_{\Theta}+N\left(1-p_{\Delta}\right)}$ scales with $p_{\Theta}^{-1} \exp \left(-\beta p_{\Theta}^{-1}\right)$, where $\beta$ is a constant.

Proof. See Appendix A.

\section{B. Coverage probability}

The following lemma derives the coverage probability under the path-loss function $\ell($.$) and the scheduling model 1$.

Lemma 2. The coverage probability under the scheduling model 1 and a path-loss function $\ell($.$) validating the depen-$ dency condition is expressed as

$$
\begin{aligned}
& \mathrm{P}_{\text {cov }}^{(1)}\left(T_{q}, T_{s}\right)=\pi \lambda_{b} p_{\Theta} \\
& \times \int_{0}^{\gamma} \exp \left\{-\pi \lambda_{b} x\left(p_{\Theta}+\left(1-p_{\Delta}\right) \int_{1}^{\infty} \frac{d y}{1+\frac{\ell(\sqrt{x y})}{T_{q} \ell(\sqrt{x})}}\right)\right\} d x,
\end{aligned}
$$

where $\gamma=\max \left(0,\left[\ell^{-1}\left(\mathrm{SNR} / T_{s}\right)\right]^{2}\right)$.

Proof. See Appendix B. 


\section{Theorem 1.}

$$
\begin{aligned}
& \mathrm{P}_{\text {cov }}^{(1)}\left(T_{q}, T_{s}\right)=p_{\Theta} \frac{1-\exp \left(-\pi \lambda_{b} \mathcal{A} \mathcal{Q}\left(\lambda_{u}, \lambda_{b}, \delta, T_{q}\right)\right)}{\mathcal{Q}\left(\lambda_{u}, \lambda_{b}, \delta, T_{q}\right)} \exp \left(-\pi \lambda_{b} h^{2}\left(\mathcal{Q}\left(\lambda_{u}, \lambda_{b}, \delta, T_{q}\right)-p_{\Theta}\right)\right), \\
& \mathrm{P}_{\text {cov }}^{(2)}\left(T_{q}, T_{s}\right)=\frac{\lambda_{b}}{\lambda_{u}}\left(1-p_{\Delta}\right) \mathrm{P}_{\text {cov }}^{(1)}\left(T_{q}, T_{s}\right), \quad \mathrm{P}_{\text {cov }}^{(3)}\left(T_{q}, T_{s}\right)=\sum_{n=0}^{\infty} \mathbb{P}\left\{\mathcal{N}_{u}=n\right\}\left[\sum_{k=1}^{n+1}\left(\begin{array}{c}
n+1 \\
k
\end{array}\right)(-1)^{k+1} \mathrm{P}_{\text {cov }}^{(1)}\left(k T_{q}, T_{s}\right)\right] \\
& \text { where } \mathcal{Q}\left(\lambda_{u}, \lambda_{b}, m, T_{q}\right)=p_{\Theta}+\left(1-p_{\Delta}\right)\left(F_{m}\left(T_{q}\right)-1\right), \text { and } \mathcal{A}=\max \left(0,\left[\left(\frac{\mathrm{SNR}}{T_{s}}\right)^{\delta}-h^{2}\right]\right) .
\end{aligned}
$$

Proof. See Appendix C.

For the remainder, we adopt the standard power-law pathloss model with elevated BSs of height $h \geq 0$, i.e., $\ell\left(r_{k}\right)=$ $\left(r_{k}^{2}+h^{2}\right)^{\frac{\alpha}{2}}$ for all $x_{k} \in \Psi_{\mathrm{b}}$, where $\alpha$ is the path-loss exponent assumed to be $\alpha>2$, and let $\delta=2 / \alpha$. Generalizing our work to other relevant path-loss models such as the dual-slope [6] and stretched path-loss models, is left to future works.

The following theorem in the top of this page, gives the coverage probability under the considered path-loss model and the three scheduling models.

Remark 2. The main strength of Theorem 1 is that the expressions of $\mathrm{P}_{\text {cov }}^{(1)}$ and $\mathrm{P}_{\text {cov }}^{(2)}$ are written under closed-form expressions, which is very helpful for performance evaluation as it reduces computational complexity and time-consuming processing. Moreover, the expression of $\mathrm{P}_{\text {cov }}^{(3)}$ is an infinite sum that can be further simplified by considering the assumption $\mathbb{P}\left\{\mathcal{N}_{u}=n_{0}\right\}=1$, where $n_{0}=\left\lceil\mathbb{E}\left\{\mathcal{N}_{u}\right\}\right\rceil$ and $\mathbb{E}\left\{\mathcal{N}_{u}\right\}=$ $1+1.28\left(\lambda_{u} / \lambda_{b}\right)$ [15, Lemma 4]. The coverage probability under the scheduling model 3 is then approximated as

$$
\mathrm{P}_{\text {cov }}^{(3)}\left(T_{q}, T_{s}\right)=\sum_{k=1}^{n_{0}+1}\left(\begin{array}{c}
n_{0}+1 \\
k
\end{array}\right)(-1)^{k+1} \mathrm{P}_{\text {cov }}^{(1)}\left(k T_{q}, T_{s}\right) \text {. }
$$

The accuracy of this approximation will be discussed in Section IV.

Remark 3. Theorem 1 reveals that the coverage probability under the scheduling models 2 and 3 is based on its computation under the scheduling model 1. In other words, $\mathrm{P}_{\text {cov }}^{(2)}$ and $\mathrm{P}_{\text {cov }}^{(3)}$ can be expressed on the basis $\left(\mathrm{P}_{\text {cov }}^{(1)}\left(k T_{q}, T_{s}\right)\right)_{k=1, \ldots, n+1}$, where $n$ is an integer $\geq 1$. As a result, we can focus the analysis on the building block component $\mathrm{P}_{c o v}^{(1)}$.

Remark 4. The scaling law of $\mathrm{P}_{c o v}^{(j)}$ with $\lambda_{b}$ and $\lambda_{u}$ is completely defined. In fact, Lemma 1 determines the scaling law of $\left(\lambda_{b} / \lambda_{u}\right) p_{\Theta}\left(1-p_{\Delta}\right)$ and $(1-$ $\left.\exp \left(-\pi \lambda_{b} \mathcal{A} \mathcal{Q}\left(\lambda_{u}, \lambda_{b}, \delta, T_{q}\right)\right)\right) / \mathcal{Q}\left(\lambda_{u}, \lambda_{b}, \delta, T_{q}\right)$, while (22) confirms that $\exp \left(-\pi \lambda_{b} h^{2}\left(\mathcal{Q}\left(\lambda_{u}, \lambda_{b}, \delta, T_{q}\right)-p_{\Theta}\right)\right)$ scales with $\exp \left(-\kappa h^{2}\left(F_{\delta}\left(T_{q}\right)-1\right) \frac{\lambda_{b} \lambda_{u}}{\lambda_{b}+\lambda_{u}}\right)$, where $\kappa$ is a constant.

We now investigate the asymptotic behavior of $P_{\text {cov }}^{(j)}$, where the rationale is to understand the interplay between coverage probability and the parameters $\mathrm{P}_{\mathrm{tx}}, \lambda_{\mathrm{b}}, \lambda_{\mathrm{u}}$ and antennas height $h$, assuming that when acting on specific parameters, the others are supposed to be constant. The results are summarized in the following three propositions.
Proposition 1. The coverage probability under the considered path-loss function $\ell($.$) and the three scheduling models, tends$ towards a surely "universal outage" in the following asymptotic cases

$$
\lim _{\lambda_{u} \rightarrow \infty} \mathrm{P}_{c o v}^{(j)}\left(T_{q}, T_{s}\right)=\lim _{\mathcal{A} \rightarrow 0} \mathrm{P}_{c o v}^{(j)}\left(T_{q}, T_{s}\right)=0
$$

Proof. The proof follows by a direct inspection of Theorem 1 .

Remark 5. Proposition 1 shows that the intended BS needs to check the primary constraint $P_{t x}>h^{\alpha} T_{s} \sigma^{2}$ in order to generate the earliest samples of users or areas with a correct coverage. Moreover, it identifies the detrimental effect of increasing users density on BS resource capacity $\left(p_{\Theta} \rightarrow 0\right)$ and then on the network overall coverage probability.

Proposition 2. The coverage probability under the PF and RR schedulers converges to that of the non-orthogonal scheduler as $\lambda_{b} \rightarrow \infty$ or $\lambda_{u} \rightarrow 0$. Hence, $\mathrm{P}_{\text {cov }}^{(j)}\left(T_{q}, T_{s}\right)$ tends towards a limit conditioned on other parameters, as

$$
\begin{aligned}
& \text { i) } \lim _{\lambda_{b} \rightarrow \infty} \mathrm{P}_{c o v}^{(j)}\left(T_{q}, T_{s}\right)=\exp \left(-\pi \lambda_{u} h^{2}\left(F_{\delta}\left(T_{q}\right)-1\right)\right), \\
& \text { ii) } \lim _{\lambda_{u} \rightarrow 0} \mathrm{P}_{\text {cov }}^{(j)}\left(T_{q}, T_{s}\right)=1-\exp \left(-\pi \lambda_{b} \mathcal{A}\right) .
\end{aligned}
$$

Proof. The sketch of the proof stems from Remark 1, and the observation that

$$
\lim _{\lambda_{\mathrm{b}} \rightarrow \infty} \mathbb{P}\left\{\mathcal{N}_{\mathbf{u}}=n\right\}=\lim _{\lambda_{\mathrm{u}} \rightarrow 0} \mathbb{P}\left\{\mathcal{N}_{\mathbf{u}}=n\right\}= \begin{cases}1 & \text { if } n=0 \\ 0 & \text { if } n \geq 1\end{cases}
$$

Remark 6. Several earlier works suggested that the tendency of coverage probability in UDNs is either towards 0 [6] or 1 [16], which involves that the performance of different networks will be the same in the regime of high BS density. However, we show in (12) a more precise result, where the performance of several UDNs will be different as well as their respective users density, average BS height, path-loss exponent and the threshold $T_{q}$ are different. We note moreover from Prop. 2 that $\lim _{\lambda_{b} \rightarrow \infty} \mathrm{P}_{\text {cov }}^{(j)}\left(T_{q}, T_{s}\right)$ is independent of $T_{s}$ (more generally of $\mathrm{SNR}$ ), while $\lim _{\lambda_{u} \rightarrow 0} \mathrm{P}_{c o v}^{(j)}\left(T_{q}, T_{s}\right)$ is independent of $T_{q}$, which is due to the idle mode capability that mitigates interference as $\lambda_{b} \rightarrow \infty$ and/or $\lambda_{u} \rightarrow 0$. 
Proposition 3. Considering the reduced height and high SNR scenarios, the coverage probability under the Non-orthogonal scheduling is expressed as

$$
\begin{aligned}
& \text { i) } \lim _{h \rightarrow 0} \mathrm{P}_{c o v}^{(1)}\left(T_{q}, T_{s}\right)=p_{\Theta} \frac{1-\mathrm{e}^{-\pi \lambda_{b}\left(\frac{\mathrm{SNR}}{T_{s}}\right)^{\delta} \mathcal{Q}\left(\lambda_{u}, \lambda_{b}, \delta, T_{q}\right)}}{\mathcal{Q}\left(\lambda_{u}, \lambda_{b}, \delta, T_{q}\right)} \\
& \text { ii) } \lim _{\mathrm{SNR} \rightarrow \infty} \mathrm{P}_{c o v}^{(1)}\left(T_{q}, T_{s}\right)=p_{\Theta} \frac{\mathrm{e}^{-\pi \lambda_{b} h^{2}\left(\mathcal{Q}\left(\lambda_{u}, \lambda_{b}, \delta, T_{q}\right)-p_{\Theta}\right)}}{\mathcal{Q}\left(\lambda_{u}, \lambda_{b}, \delta, T_{q}\right)}
\end{aligned}
$$

Proof. The sketch of the proof follows by a direct inspection of Theorem 1.

We now assume that we have previously identified the parameters $T_{\mathrm{q}}$ and $T_{\mathrm{s}}$ required to access the system, the parameter $\alpha$ defined by the propagation environment, the parameter $\lambda_{\mathrm{u}}$ expected from end-users behavior. The following proposition gives the correlation constraint that needs to be established between the pre-defined parameters and the commonly used parameters for cellular network optimization, namely $\lambda_{\mathrm{b}}, h$ and $P_{\mathrm{tx}}$, in order to meet a given coverage probability $0<P^{(1)}<1$ under the baseline scheduling model.

Corollary 1. For pre-defined parameters $T_{q}, T_{s}, \lambda_{u}$ and $\delta$, the following constraint needs to be verified to meet a required coverage probability $0<P^{(1)}<1$ under the Non-orthogonal scheduling model,

$$
P^{(1)} \leq \frac{p_{\Theta}}{\mathcal{Q}\left(\lambda_{u}, \lambda_{b}, \delta, T_{q}\right)} \varrho\left(\lambda_{b}, h, P_{t x}\right),
$$

$$
\begin{aligned}
& \text { where } \varrho\left(\lambda_{b}, h, P_{t x}\right)= \\
& \min \left(e^{-\pi \lambda_{b} h^{2}\left(\mathcal{Q}\left(\lambda_{u}, \lambda_{b}, \delta, T_{q}\right)-p_{\Theta}\right)} ; 1-e^{-\pi \lambda_{b} \mathcal{A} \mathcal{Q}\left(\lambda_{u}, \lambda_{b}, \delta, T_{q}\right)}\right) .
\end{aligned}
$$

Proof. The skecth of the proof is as follows: For fixed parameters $T_{\mathrm{q}}, T_{\mathrm{s}}, \lambda_{\mathrm{u}}, \delta$ and coverage probability $P^{(1)}$. Equation (9) can be expressed as $\mathrm{P}_{\text {cov }}^{(1)}\left(T_{\mathrm{q}}, T_{\mathrm{s}}\right)=u\left(\lambda_{\mathrm{b}}\right) e^{-v\left(\lambda_{\mathrm{b}}, h\right)}(1-$ $\left.e^{-w\left(\lambda_{\mathrm{b}}, h, P_{\mathrm{lx}}\right)}\right)$, where $u\left(\lambda_{\mathrm{b}}\right)=p_{\Theta}\left(\mathcal{Q}\left(\lambda_{\mathrm{u}}, \lambda_{\mathrm{b}}, \delta, T_{\mathrm{q}}\right)\right)^{-1}$, $v\left(\lambda_{\mathrm{b}}, h\right)=\pi \lambda_{\mathrm{b}} h^{2}\left(\mathcal{Q}\left(\lambda_{\mathrm{u}}, \lambda_{\mathrm{b}}, \delta, T_{\mathrm{q}}\right)-p_{\Theta}\right)$ and $w\left(\lambda_{\mathrm{b}}, h, P_{\mathrm{tx}}\right)=$ $\pi \lambda_{\mathrm{b}} \mathcal{A} \mathcal{Q}\left(\lambda_{\mathrm{u}}, \lambda_{\mathrm{b}}, \delta, T_{\mathrm{q}}\right)$. However, since $\mathcal{A}>0$ and $T_{\mathrm{q}} \mapsto$ $F_{\delta}\left(T_{\mathrm{q}}\right)$ is a monotonically increasing function with $T_{\mathrm{q}} \geq 0$ and $0 \leq \delta<1$, where $F_{\delta}(0)=F_{0}\left(T_{\mathrm{q}}\right)=1$; the functions $u, v$ and $w$ are then positive for every parameters $\lambda_{\mathrm{b}}, h$ and $P_{\mathrm{tx}}$. The constraint (16) is then true for $P^{(1)}$ chosen such as $0<e^{-v\left(\lambda_{\mathrm{b}}, h\right)}<1$ and $0<e^{-w\left(\lambda_{\mathrm{b}}, h, P_{\mathrm{tx}}\right)}<1$ in (9), which completes the proof.

Remark 7. A direct inspection of (16) confirms that the achievable coverage probability will be maximized if the following intuitive adjustments are established: On the one hand: increase the ratio $\lambda_{b} / \lambda_{u} \gg 1, P_{t x}$ and $\alpha$. On the other hand, decrease $h, \sigma^{2}, T_{q}$ and $T_{s}$.

\section{Average Achievable Rate}

We also investigate the mean data rate achievable over a cell in units of nats $/ \mathrm{Hz}$, and verify how it is impacted with network parameters when considering the three scheduling models. Consistently with our adopted model of coverage probability
(4) [8], we introduce the following definition of the ergodic rate of a typical user associated to $x_{0}$,

$$
\begin{aligned}
\tau_{c}^{(j)}(.) & =\mathbb{E}\left\{\mathbb{1}\left\{\ell\left(r_{0}\right) \leq \frac{\mathrm{SNR}}{T_{\mathrm{s}}}\right\} \ln (1+\mathrm{SIR})\right\} \\
& \stackrel{(\mathrm{a})}{=} \int_{t>0} \mathrm{P}_{\mathrm{cov}}^{(j)}\left(\mathrm{e}^{t}-1, T_{\mathrm{s}}\right) \mathrm{d} t,
\end{aligned}
$$

where $j$ refers to the scheduling model, and (a) follows from similar steps in [3, Theorem 3].

We have then; from Theorem 1 and some variable changes,

$$
\begin{aligned}
& \tau_{c}^{(2)}(.)=\frac{\lambda_{\mathrm{b}}}{\lambda_{\mathrm{u}}}\left(1-p_{\Delta}\right) \tau_{c}^{(1)}(.), \\
& \tau_{c}^{(3)}=\sum_{n=0}^{\infty} \mathbb{P}\left\{\mathcal{N}_{\mathrm{u}}=n\right\}\left[\sum_{k=1}^{n+1}\left(\begin{array}{c}
n+1 \\
k
\end{array}\right)(-1)^{k+1} \int_{x>0} \frac{\mathrm{P}_{\mathrm{cov}}^{(1)}\left(k x, T_{\mathrm{s}}\right)}{\mathrm{d} x}\right] .
\end{aligned}
$$

Note that the expression of ergodic rate in [3, Theorem 3], needs generally, the computation of a four-fold numerical integral, whereas the expression in (18), requires only the computation of a two-fold integral, which is expected to be more computationally efficient.

\section{Numerical Results}

In this section, we present numerical results to validate our theoretical analysis and assess the network performance trend as a function of several key parameters adjustment. We consider in the following, $\sigma^{2}=0 \mathrm{~dB}, \alpha=4, T_{\mathrm{s}}$ is mapped with $T_{\mathrm{q}}$ by the expression $T_{\mathrm{s}}=T_{\mathrm{q}} \Gamma(1+\delta)^{-1 / \delta}[9$, Eq. (5)], where $\Gamma($.$) denotes the complete Gamma function.$ Integral expressions are evaluated using Matlab, and Monte Carlo simulations are performed with $10^{4}$ iterations.

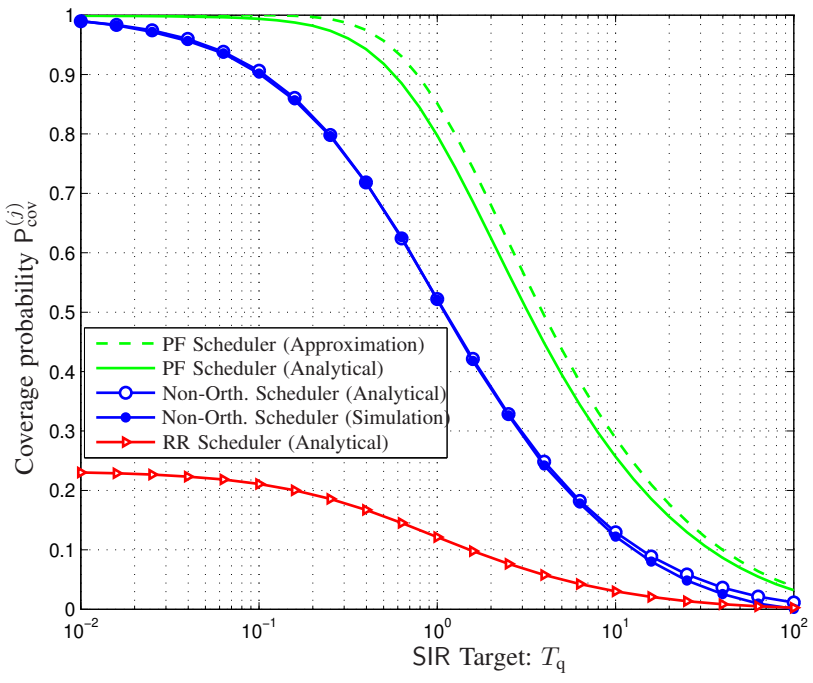

Fig. 2. The scaling of coverage probability $\mathrm{P}_{\text {cov }}^{(j)}$ with SIR target $T_{\mathrm{q}}$, for $\lambda_{\mathrm{b}}=\lambda_{\mathrm{u}} / 4=0.25 \mathrm{BS} / \mathrm{m}^{2}, \eta=10^{3}, h=0.4 \mathrm{~m}$, and $P_{\mathrm{tx}}=43 \mathrm{dBm}$.

Fig. 2 shows that the simulation results of coverage probability under the baseline scheduling model, match perfectly with the analytical expression, which validates the accuracy of our analytical model. Moreover, and consistently with previous results considering the conventional definition of coverage 


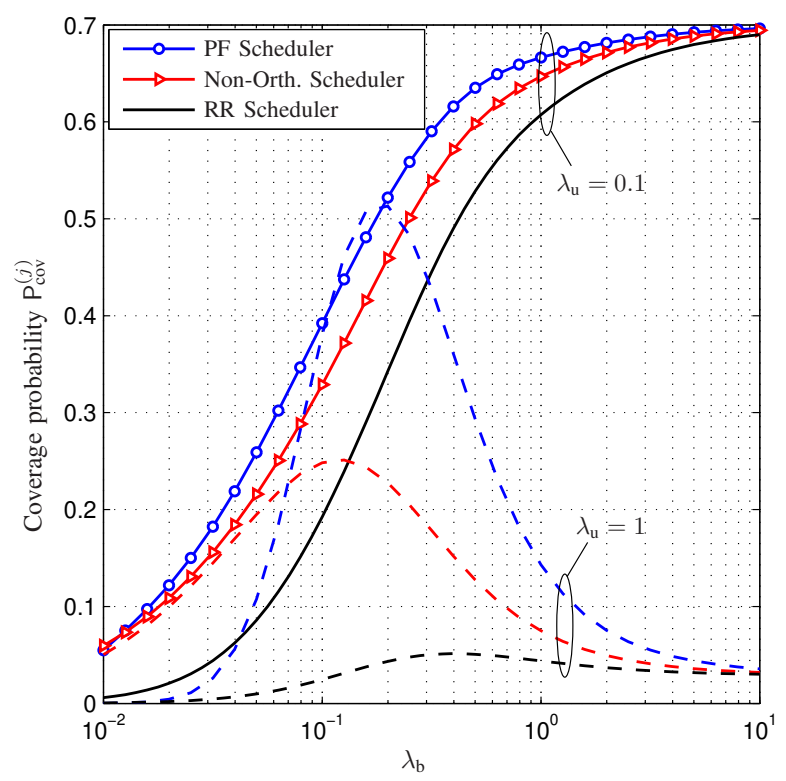

Fig. 3. The scaling of coverage probability $\mathrm{P}_{\text {cov }}^{(j)}$ with network density $\lambda_{\mathrm{b}}$ and users density $\lambda_{\mathrm{u}}$. The other parameters are fixed as: $P_{\mathrm{tx}}=43 \mathrm{dBm}$, $T_{\mathrm{q}}=2 \mathrm{~dB}, \eta=10^{3}, h=1 \mathrm{~m}$.

probability [12], Fig. 2 shows that under the new coverage probability framework [8], the PF scheduler is the model that best improves the coverage probability, due to the multi-user diversity gain. However, the RR scheduler [11], reduces the coverage probability due to the equal probability scheduling process, particularly for large users density. Besides, Fig. 2 shows that the approximation expression (11), is generally a sufficient tight upper-bound of $\mathrm{P}_{\mathrm{cov}}^{(3)}$, reflecting the main trends of $\mathrm{P}_{\text {cov }}^{(3)}$.

Fig. 3 shows that for constant $P_{\mathrm{tx}}, T_{\mathrm{q}}, \eta$ and $h$, the behavior of coverage probability in UDNs scales with user and BS densities, $\lambda_{\mathrm{u}}$ and $\lambda_{\mathrm{b}}$, respectively. More generally, for large $\lambda_{\mathrm{b}}$, the coverage probability under the three representative scheduling schemes scales with $\exp \left(-\pi \lambda_{\mathrm{u}} h^{2}\left(F_{\delta}\left(T_{\mathrm{q}}\right)-1\right)\right)$ consistently with Eq. (12).

Fig. 4 shows the scaling trend of $\mathrm{P}_{\text {cov }}^{(j)}$ with BS density $\lambda_{\mathrm{b}}$ as a function of $\eta, P_{\mathrm{tx}}$ and $h$. The figure confirms Proposition 2, where the three scheduling models are equivalent in UDNs. Consequently, we recommend to deploy a scheduling strategy with the most reduced implementation complexity in UDNs. Moreover, we mention the limited impact of inherent resource capacity $\eta$ and the BS transmit power $P_{\mathrm{tx}}$ as network density increases. In other words, $\eta$ and $P_{\mathrm{tx}}$ need to scale with nearly a $1 / \lambda_{\mathrm{b}}$ rate to reduce infrastructure power consumption and the cost of acquired resource capacity. In this context, investigating the optimum scaling law of $P_{\mathrm{tx}}$ and $\eta$ with $\lambda_{\mathrm{b}}$ as part of an energy efficiency setup, in addition to approximating the BS density that maximizes the network performance will be left to future work.

Fig. 5 evaluates expression (18), where it confirms the detrimental effect of BS height $h$ and users density $\lambda_{\mathrm{u}}$ on the network average rate. In addition, and in agreement with our previous results, we conclude the limited impact of inherent resource capacity $\eta$ and the transmit power $P_{\mathrm{tx}}$ as $\lambda_{\mathrm{b}}$ increases.

\section{CONCLUSION}

Considering a revisited version of the coverage probability recent definition, introduced in [8], this paper derived the coverage probability and the average data rate for a downlink cellular network with elevated BSs and three representative scheduling models. The network performance under a given scheduling model is shown to be expressed on the basis of that under non-orthogonal scheduling, where competing users are served on the same resource block. In addition, it is revealed that the PF scheduler gives the best network performance due to multi-user diversity gain, while the RR scheduling is impaired by users density due to the equal probability selection process. However, the three scheduling models are equivalent in the context of UDNs, where we recommend to deploy the scheduling model with the most reduced implementation complexity.

Besides, our results showed that BS height and user density are so detrimental to coverage probability and average rate in UDNs, while inherent resource capacity and the transmit power have reduced impact as network density increases, which suggests new insights into the role of these parameters in UDNs.

\section{APPENDIX A}

PROOF OF LEMMA 1

For a given users density $\lambda_{\mathrm{u}} \geq 0$ and a real $q>1, \exists \lambda_{\mathrm{b}}^{0}=$ $\frac{1}{q-1} \lambda_{\mathrm{u}}$ such as: $\forall \lambda_{\mathrm{b}}>\lambda_{\mathrm{b}}^{0}, p_{\Theta}^{-1} \leq\left(1+\frac{q \lambda_{\mathrm{u}}}{\eta\left(\lambda_{\mathrm{b}}+\lambda_{\mathrm{u}}\right)}\right)^{2}$. Using the observation that $\exp (-x)<\left(1+\frac{x}{y}\right)^{-y}<\exp \left(\frac{-x y}{x+y}\right)$ for every reals $x, y>0$, we get

$$
\begin{aligned}
& \exp \left(\frac{-2 q \lambda_{\mathrm{u}}}{\eta\left(\lambda_{\mathrm{b}}+\lambda_{\mathrm{u}}\right)}\right) \leq p_{\Theta} \leq \exp \left(\frac{-2 \lambda_{\mathrm{u}}}{\max (1, \eta)\left(\lambda_{\mathrm{b}}+\lambda_{\mathrm{u}}\right)}\right), \\
& 1-\exp \left(\frac{-\lambda_{\mathrm{u}}}{\lambda_{\mathrm{b}}+\lambda_{\mathrm{u}}}\right) \leq 1-p_{\triangle} \leq 1-\exp \left(\frac{-\lambda_{\mathrm{u}}}{\lambda_{\mathrm{b}}}\right)
\end{aligned}
$$

Moreover, using the observation that $\exists 0<m<1$ such as for every real $x>0$

$\exp \left(-\frac{1}{x}\right)<\frac{x}{x+1}<1-\exp (-x)<\exp \left(\frac{-m}{x}\right)<\frac{x}{m(x+1)}$,

we then get,

$$
\frac{\lambda_{\mathrm{u}}}{2\left(\lambda_{\mathrm{b}}+\lambda_{\mathrm{u}}\right)} \leq 1-p_{\triangle} \leq \frac{\lambda_{\mathrm{u}}}{m\left(\lambda_{\mathrm{b}}+\lambda_{\mathrm{u}}\right)} .
$$

The proof of i) is then completed by combining (19) and (22).

For every real $M$ and $N \geq 0$, we have $p_{\Theta} \leq p_{\Theta}+N\left(1-p_{\Delta}\right) \leq N+(N+1) p_{\Theta}$, and by applying (21), we can see that for a given users density $\lambda_{\mathrm{u}}$, $\exists \lambda_{\mathrm{b}}^{1}=\lambda_{\mathrm{u}} /(\eta[\sqrt{(N+1) / N}-1])$ such as: $\forall \lambda_{\mathrm{b}} \geq \lambda_{\mathrm{b}}^{1}$, $\left(1-\exp \left(-M\left[p_{\Theta}+N\left(1-p_{\Delta}\right)\right]\right)\right) /\left[p_{\Theta}+N\left(1-p_{\Delta}\right)\right]$

is bounded between $\frac{1}{2(N+1)} p_{\Theta}^{-1} \exp \left(-\frac{p_{\Theta}^{-1}}{M}\right)$ and $p_{\Theta}^{-1} \exp \left(-\left(m p_{\Theta}^{-1} /[2 M(N+1)]\right)\right)$, which completes the proof of (ii). 


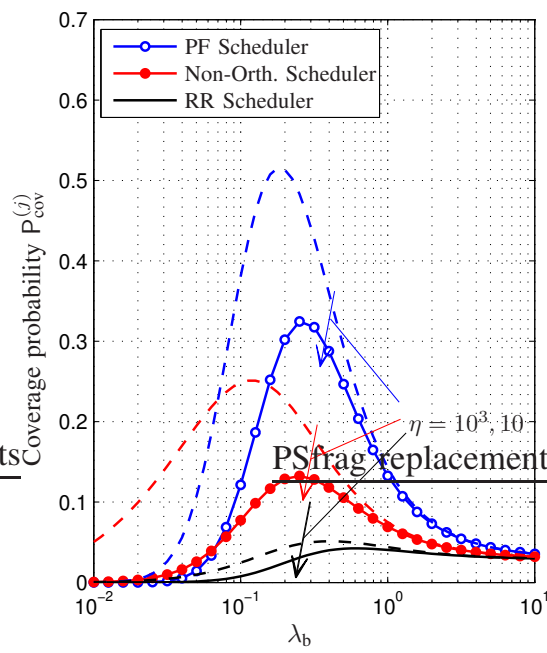

(a)

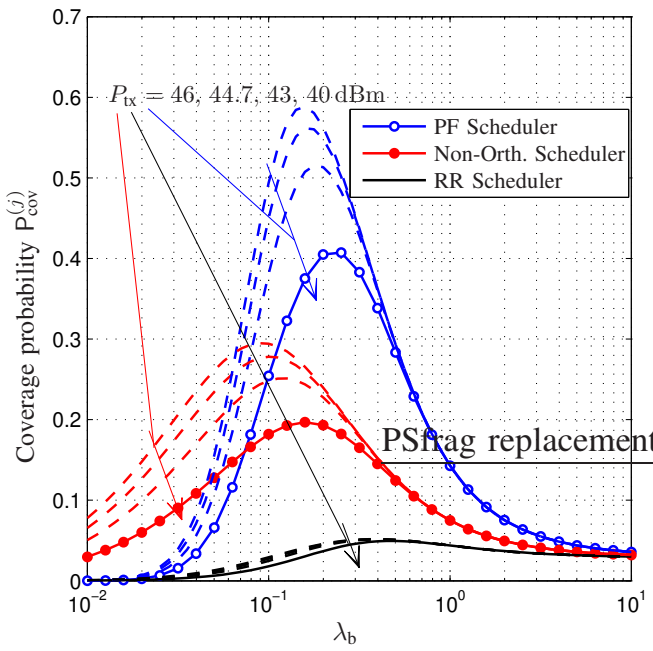

(b)

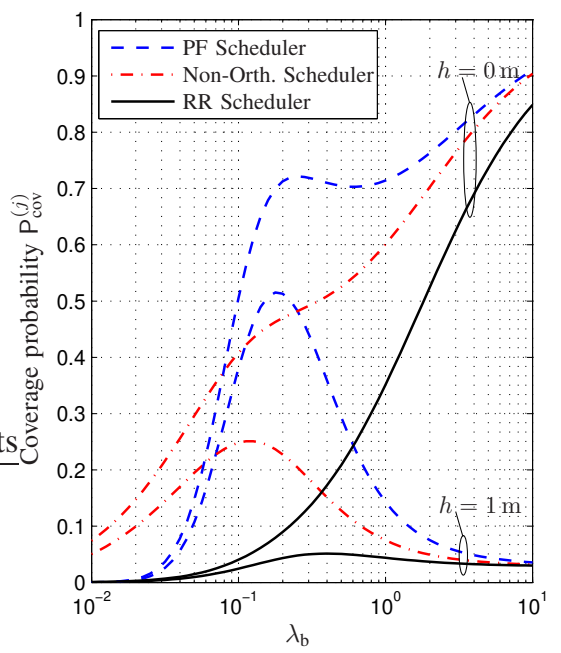

(c)

Fig. 4. For $\lambda_{\mathrm{u}}=1 \mathrm{user} / \mathrm{m}^{2}$ and $T_{\mathrm{q}}=2 \mathrm{~dB}$, we plot the scaling of coverage probability $\mathrm{P}_{\mathrm{cov}}^{(j)}$ with network density $\lambda_{\mathrm{b}}$ as a function of: (a) The resource capacity $\eta$ when $P_{\mathrm{tx}}=43 \mathrm{dBm}, h=1 \mathrm{~m}$, (b) The transmit power $P_{\mathrm{tx}}$ when $\eta=10^{3}$ and $h=1 \mathrm{~m}$, (c) The BS height $h$ when $\eta=10^{3}$ and $P_{\mathrm{tx}}=43 \mathrm{dBm}$.

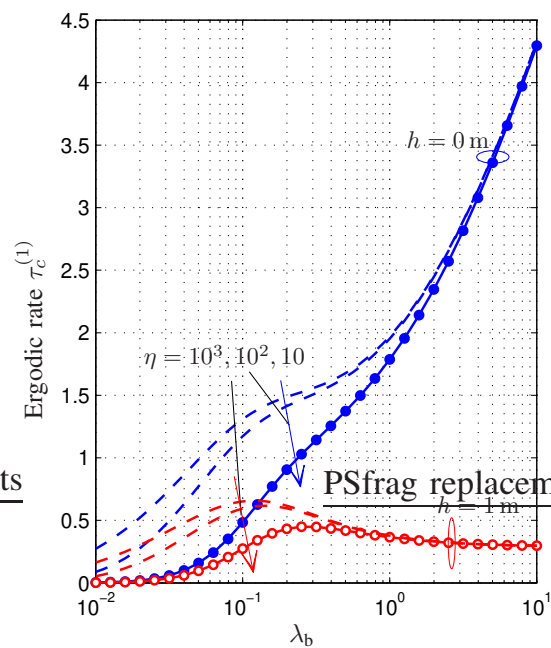

(a)

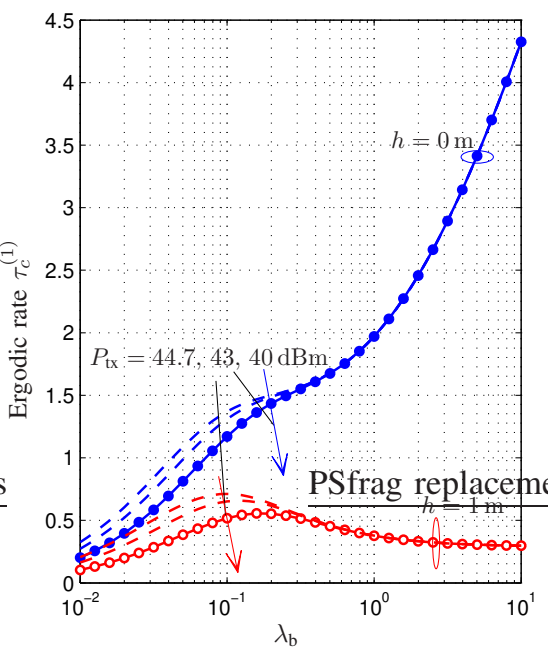

(b)

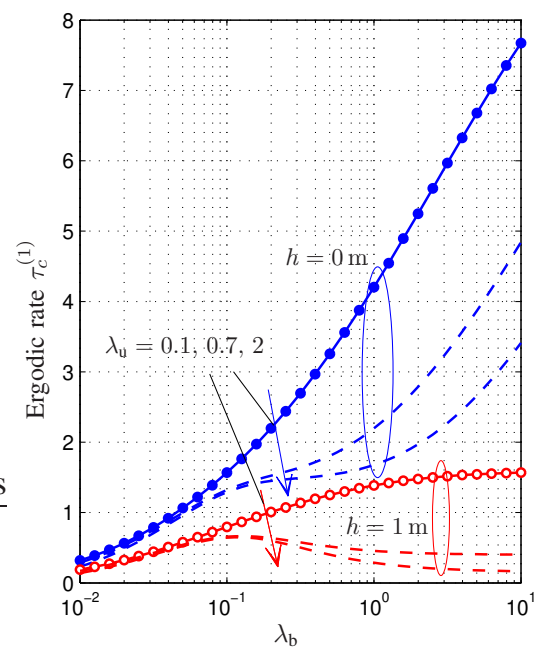

(c)

Fig. 5. The scaling of the average rate under the non-orthogonal scheduling, $\tau_{c}^{(1)}$, with network density $\lambda_{\mathrm{b}}$, as a function of: (a) The resource capacity $\eta$ and BS height $h$ when $\lambda_{\mathrm{u}}=1$ user $/ \mathrm{m}^{2}$ and $P_{\mathrm{tx}}=43 \mathrm{dBm}$, (b) The transmit power $P_{\mathrm{tx}}$ and BS height $h$ when $\lambda_{\mathrm{u}}=1$ user $/ \mathrm{m}^{2}$ and $\eta=10^{3}$, (c) The user density $\lambda_{\mathrm{u}}$ and BS height $h$ when $\eta=10^{3}$ and $P_{\mathrm{tx}}=43 \mathrm{dBm}$.

\section{APPENDIX B}

PROOF OF LEMMA 2

The coverage probability in (4), is expressed under the scheduling model 1 as:

$$
\begin{aligned}
& \mathrm{P}_{\text {cov }}^{(1)}\left(T_{\mathrm{q}}, T_{\mathrm{s}}\right)=\mathbb{P}\left\{\mathrm{SIR} \geq T_{\mathrm{q}}, \overline{\mathrm{SNR}} \geq T_{\mathrm{s}}, \mathcal{N}_{\mathrm{u}}=0\right\} \\
& =2 \pi \lambda_{\Theta} \int_{0}^{\infty} \mathrm{e}^{-\pi \lambda_{\Theta} r^{2}} \mathbb{E}_{I}\left\{\mathbb{P}\left\{\mathrm{SIR} \geq T_{\mathrm{q}}, \overline{\mathrm{SNR}} \geq T_{\mathrm{s}} \mid I, r\right\}\right\} r \mathrm{~d} r \\
& \stackrel{(\mathrm{a})}{=} 2 \pi \lambda_{\Theta} \int_{0}^{\infty} \mathrm{e}^{-\pi \lambda_{\Theta} r^{2}} \mathbb{1}\left\{\ell(r) \leq \frac{\mathrm{SNR}}{T_{\mathrm{s}}}\right\} \mathbb{E}_{I}\left\{\mathbb{P}\left\{\mathrm{SIR} \geq T_{\mathrm{q}} \mid I, r\right\}\right\} r \mathrm{~d} r \\
& =2 \pi \lambda_{\Theta} \int_{0}^{\sqrt{\gamma}} \mathrm{e}^{-\pi \lambda_{\Theta} r^{2}} \underbrace{\mathbb{E}_{I}\left\{\mathbb{P}\left\{\mathrm{SIR} \geq T_{\mathrm{q}} \mid I, r\right\}\right\}}_{\chi\left(T_{\mathrm{q}}, T_{\mathrm{s}}\right)} r \mathrm{~d} r,
\end{aligned}
$$

and the expectation term, $\chi\left(T_{\mathrm{q}}, T_{\mathrm{s}}\right)$, in the integrand function, can be computed as follows:

$$
\begin{aligned}
& \chi\left(T_{\mathrm{q}}, T_{\mathrm{s}}\right)=\mathbb{L}_{I}\left\{\frac{T_{\mathrm{q}} \ell(r)}{P_{\mathrm{tx}}}\right\}=\mathbb{E}_{\Psi_{\mathrm{b}} \backslash \Delta,\left\{g_{i}\right\}}\left\{\prod_{x_{i} \in \Psi_{\mathrm{b}} \backslash \Delta} \frac{\ell\left(r_{i}\right)}{\ell\left(r_{i}\right)+T_{\mathrm{q}} \ell(r)}\right\} \\
& \stackrel{(\mathrm{b})}{=} \exp \left(-2 \pi\left(\lambda_{\mathrm{b}}-\lambda_{\Delta}\right) \int_{r}^{\infty} \frac{T_{\mathrm{q}} \ell(r) u \mathrm{~d} u}{\ell(u)+T_{\mathrm{q}} \ell(r)}\right) \\
& \stackrel{(\mathrm{c})}{=} \exp \left(-\pi \lambda_{\mathrm{b}}\left(1-p_{\Delta}\right) r^{2} \int_{1}^{\infty} \frac{\mathrm{d} u}{1+\frac{\ell(r \sqrt{u})}{T_{\mathrm{q}} \ell(r)}}\right)
\end{aligned}
$$

where (a) follows from the independence of $\overline{\mathrm{SNR}}$ from the cumulative other-cell interference $I$. (b) follows from the PGFL theorem and (c) from the variable change $u^{2} / r^{2} \rightarrow u$. Plugging (24) into (23) with $r^{2} \rightarrow x$ gives the desired result. 


\section{APPENDIX C \\ PROOF OF THEOREM 1}

The proof of the expression of $\mathrm{P}_{\mathrm{cov}}^{(1)}$ in Theorem 1 follows directly from Lemma 2 where $\gamma=\mathcal{A}^{2}$, and (24) is derived as

$$
\begin{aligned}
& \chi\left(T_{\mathrm{q}}, T_{\mathrm{s}}\right)=\exp \left(-\pi \lambda_{\mathrm{b}}\left(1-p_{\Delta}\right)\left(r^{2}+h^{2}\right) T_{\mathrm{q}}^{\frac{2}{\alpha}} \int_{T_{\mathrm{q}}-\frac{2}{\alpha}}^{\infty} \frac{\mathrm{d} u}{1+u^{\frac{\alpha}{2}}}\right) \\
& \stackrel{(\mathrm{a})}{=} \mathrm{e}^{\left(-\pi \lambda_{\mathrm{b}}\left(1-p_{\Delta}\right)\left(F_{\delta}\left(T_{\mathrm{q}}\right)-1\right) r^{2}\right)} \mathrm{e}^{\left(-\pi \lambda_{\mathrm{b}}\left(1-p_{\Delta}\right)\left(F_{\delta}\left(T_{\mathrm{q}}\right)-1\right) h^{2}\right)},
\end{aligned}
$$

where (a) holds since $T_{\mathrm{q}}^{\frac{2}{\alpha}} \int_{T_{\mathrm{q}}^{-}}^{\infty} \frac{2}{\alpha} \frac{\mathrm{d} u}{1+u^{\frac{\alpha}{2}}}+1=F_{\delta}\left(T_{\mathrm{q}}\right)$.

The coverage probability under the scheduling model 2 , $\mathrm{P}_{\mathrm{cov}}^{(2)}$, is expressed as

$$
\begin{aligned}
& \mathrm{P}_{\mathrm{cov}}^{(2)}\left(T_{\mathrm{q}}, T_{\mathrm{s}}\right)=\sum_{n=0}^{\infty} \mathbb{P}\left\{\mathrm{SIR} \geq T_{\mathrm{q}}, \overline{\mathrm{SNR}} \geq T_{\mathrm{s}}, \mathcal{N}_{\mathrm{u}}=n\right\} \\
& =\sum_{n=0}^{\infty} \mathbb{P}\left\{\mathrm{SIR} \geq T_{\mathrm{q}}, \overline{\mathrm{SNR}} \geq T_{\mathrm{s}} \mid \mathcal{N}_{\mathrm{u}}=n\right\} \mathbb{P}\left\{\mathcal{N}_{\mathrm{u}}=k\right\} \\
& \stackrel{(\mathrm{a})}{=} \mathbb{P}\left\{\mathrm{SIR} \geq T_{\mathrm{q}}, \overline{\mathrm{SNR}} \geq T_{\mathrm{s}}\right\} \sum_{k=0}^{\infty} \frac{\mathbb{P}\left\{\mathcal{N}_{\mathrm{u}}=n\right\}}{k+1} \\
& \stackrel{(\mathrm{b})}{=} \frac{\lambda_{\mathrm{b}}}{\lambda_{\mathrm{u}}}\left(1-p_{\Delta}\right) \mathbb{P}\left\{\mathrm{SIR} \geq T_{\mathrm{q}}, \widetilde{\mathrm{SNR}} \geq T_{\mathrm{s}}\right\},
\end{aligned}
$$

where (a) follows from the independence of the events $\mathcal{E}=\left(\mathrm{SIR} \geq T_{\mathrm{q}}, \widetilde{\mathrm{SNR}} \geq T_{\mathrm{s}}\right)$ and $\mathcal{F}_{n}=\left\{\mathcal{N}_{\mathrm{u}}=n\right\}$, in addition to the properties of the RR scheduling such as each user is selected with the same probability. (b) follows from [11, Proposition 2] where $\sum_{n=0}^{\infty}(n+1)^{-1} \mathbb{P}\left(\mathcal{N}_{\mathrm{u}}=n\right)=\left(\lambda_{\mathrm{b}} / \lambda_{\mathrm{u}}\right)\left(1-p_{\Delta}\right)$.

The coverage probability under the scheduling model 3 , $P_{\mathrm{cov}}^{(3)}$, is expressed from (25) as

$$
\begin{aligned}
& \mathrm{P}_{\text {cov }}^{(3)}\left(T_{\mathrm{q}}, T_{\mathrm{s}}\right)= \\
& \sum_{n=0}^{\infty} \underbrace{\mathbb{P}\left\{\frac{\max \left(g_{0}, \ldots, g_{n}\right) P_{\mathrm{tx}}}{\ell\left(r_{0}\right) I} \geq T_{\mathrm{q}}, \overline{\mathrm{SNR}} \geq T_{\mathrm{s}}\right\}}_{\varphi\left(T_{\mathrm{q}}, T_{\mathrm{s}}\right)} \mathbb{P}\left\{\mathcal{N}_{\mathrm{u}}=n\right\},
\end{aligned}
$$

where the probability term, $\varphi\left(T_{\mathrm{q}}, T_{\mathrm{s}}\right)$, is derived as

$$
\begin{aligned}
& \varphi\left(T_{\mathrm{q}}, T_{\mathrm{s}}\right)= \\
& \mathbb{E}_{r_{0}, I}\left\{\mathbb{P}\left\{\max \left(g_{0}, \ldots, g_{n}\right) \geq \frac{T_{\mathrm{q}} \ell\left(r_{0}\right) I}{P_{\mathrm{tx}}}, \overline{\mathrm{SNR}} \geq T_{\mathrm{s}} \mid I, r_{0}\right\}\right\} \\
& \stackrel{(\text { a) }}{=} \mathbb{E}_{r_{0}, I}\left\{\mathbb{1}\left\{\ell\left(r_{0}\right) \leq \frac{\mathrm{SNR}}{T_{\mathrm{s}}}\right\}\left[1-\left(1-\exp \left(-\frac{T_{\mathrm{q}} \ell\left(r_{0}\right) I}{P_{\mathrm{tx}}}\right)\right)^{n+1}\right]\right\} \\
& \stackrel{\text { (b) }}{=} \sum_{k=1}^{n+1}\left(\begin{array}{c}
n+1 \\
k
\end{array}\right)(-1)^{k+1} \mathbb{E}_{r_{0}}\left\{\mathbb{1}\left\{\ell\left(r_{0}\right) \leq \frac{\mathrm{SNR}}{T_{\mathrm{s}}}\right\} \mathbb{L}_{I}\left\{-\frac{k T_{\mathrm{q}} \ell\left(r_{0}\right)}{P_{\mathrm{tx}}}\right\}\right\},
\end{aligned}
$$

where (a) holds since the random variables $g_{k}, k=0, \ldots, n$ are i.i.d. exponential RVs with mean 1 , and the cumulative function (CDF) of $\max \left(g_{0}, \ldots, g_{n}\right)$ is expressed as

$$
\mathbb{P}\left\{\max \left(g_{0}, \ldots, g_{n}\right) \leq u\right\}=\left(1-\mathrm{e}^{-u}\right)^{n+1},
$$

(b) follows from the binomial theorem. We get the desired result by combining the last expression with (23).

\section{REFERENCES}

[1] J. Andrews, "Seven ways that HetNets are a cellular paradigm shift," IEEE Commun. Mag., vol. 51, no. 3, pp. 136-144, Mar. 2013.

[2] D. López-Pérez, M. Ding, H. Claussen and A. H. Jafari, "Towards $1 \mathrm{Gbps} / \mathrm{UE}$ in cellular systems: understanding ultra-dense small cell deployments", IEEE Commun. Surveys \& Tutorials, vol. 17, no. 4, pp. 2078-2101, Fourth quarter 2015.

[3] J. Andrews, F. Baccelli, and R. Ganti, "A tractable approach to coverage and rate in cellular networks", IEEE Trans. Commun., vol. 59, no. 11, pp. 3122-3134, Nov. 2011

[4] F. Baccelli and B. Blaszczyszyn, Stochastic geometry and wireless networks, Part I: Theory, Part II: Applications. Now Publishers, 2009.

[5] H. S. Dhillon, R. K. Ganti, F. Baccelli, and J. G. Andrews, "Modeling and analysis of K-tier downlink heterogeneous cellular networks", IEEE J. Sel. Areas Commun., vol. 30, no. 3, pp. 550-560, Apr. 2012

[6] X. Zhang, and J. G. Andrews, "Downlink cellular network analysis with multi-slope path loss models", IEEE Trans. Commun., vol. 63, no. 5, pp. 1881-1894, May 2015.

[7] M. D. Renzo, A. Guidotti and G. E. Corazza, "Average rate of downlink heterogeneous cellular networks over generalized fading channels: A stochastic geometry approach", IEEE Trans. Commun., vol. 61, no. 7, pp. 3050-3071, July 2013.

[8] M. Di Renzo, A. Zappone, T. T. Lam, and M. Debbah, "System-level modeling and optimization of the energy efficiency in cellular networks - A stochastic geometry framework", IEEE Trans. Wireless Commun., vol. 17, no. 4, pp. 2539-2556, Apr. 2018.

[9] M. Di Renzo, T. T. Lam, A. Zappone and M. Debbah, "A tractable closed-form expression of the coverage probability in poisson cellular networks", IEEE Wireless Commun. Letters.

[10] M. Ding and D. Lopez Perez, "Please lower small cell antenna heights in 5G," in Proc. IEEE Global Communications Conference (GLOBECOM),pp. 1-6, 4-8 Dec. 2016, Washington, USA.

[11] S. M. Yu and S.-L. Kim, "Downlink capacity and base station density in cellular networks", IEEE Workshop on Spatial Stochastic Models for Wireless Networks (SpaSWiN), pp. 1-7, 13 May 2013. Tsukuba Science City, Japan.

[12] M. Ding, D. Lopez Perez, A. H. Jafari, G. Mao and Z. Lin, "Ultra-dense networks: A new look at the proportional fair scheduler," in Proc. IEEE Global Communications Conference (GLOBECOM), pp. 1-7, 4-8 Dec. 2017, Singapore.

[13] S. G. Foss, S. A. Zuyev, "On a Voronoi aggregative process related to a bivariate Poisson process," Adv. Appl. Probab., vol. 28, no. 4, pp. 965-981, Dec. 1996.

[14] P. Calka, "Precise formulae for the distributions of the principal geometric characteristics of the typical cells of a two-dimensional Poisson Voronoi tessellation and a Poisson line process", Adv. Appl. Prob., vol. 35, pp. 551-562, Sep. 2003.

[15] H. S. Dhillon and J. G. Andrews, "Downlink rate distribution in heterogeneous cellular networks under generalized sell Selection", IEEE Wireless Commun. Letters, vol. 3, no. 1, pp. 42-45, Feb. 2014.

[16] M. Ding, D. L. Perez, G. Mao, and Z. Lin, "Study on the idle mode capability with LOS and NLOS transmissions", in Proc. IEEE Global Communications Conference (GLOBECOM), pp. 16, Dec. 2016, Washington, USA. 